\title{
Toward EFFiCient NeTWork ResourCe SHaring: From One-Sided Market to Two-Sided Market
}

\author{
Yanru Zhang, Yingjie Zhou, Zhi Liu, Bidushi Barua, and Duy H. N. Nguyen
}

\section{ABSTRACT}

Resource sharing in wireless networks has been a hot topic for years. It mainly deals with two main goals: incentive mechanism design to motivate resource owners to contribute resources on the supply side of the market, and resource allocation to efficiently assign the obtained resources to end users on the demand side. Mos existing resource sharing is based on the one-sided model. However, one application of the two-sided model, sharing economy, is reshaping conventional business models with a substantial growth in both market size and profit. We see a great potential to increase profit and efficiency by adopting the two-sided market model in wireless networks, so as to bridge user demand and resource supply simultaneously from both sides of the network. However, as to wireless networks with typical network features, many problems cannot be tackled based upon existing models. In this work, we provide the basic concept of a two-sided market, together with the challenges and applications of using a two-sided market model to tackle the resource sharing problem in various kinds of networks. Potential methodologies to solve resource sharing problems in two-sided markets are also presented sequentially and compared. In the end, future directions for resource sharing under the two-sided market model are discussed.

\section{INTRODUCTION}

There is an urgent need for massive capacity to support a wide range of broadband services with the ongoing development of wireless networks. The shortage of network capacity tends to become more complicated with the evolution of mobile technologies during the deployment of $5 \mathrm{G}$ networks. Facing such a shortage of network resources and the high cost of capital expenditure, the idea of resource sharing has received considerable attention from regulators around the world, as it may create new opportunities for network operators to exploit idle resources whenever they are underutilized by their owners. Such kind of resource sharing can be widely found in areas such as spectrum sharing in cognitive radio networks, network access point sharing in cellular networks, power line sharing in smart grid, and and so on. Through this sharing, idle resources can be efficiently utilized and allocated among end users by network operators in the systems.
Successful sharing of network resources mainly involves two interconnected goals: motivating owners to contribute resources and efficiently allocating the resources to end users. The network operator achieves the first objective through incentive mechanism designs to efficiently motivate resource owners and provide the maximum amount of resources. The second objective is to assign and manage the obtained resources in an efficient manner that supports the system's strategic goals, such as maximizing profit or efficiency. However, instead of solving the resource sharing problems by considering both sides of the market simultaneously, as far as the authors understand, most of works in literature study the two objectives separately based on one-sided market models as shown in Fig. 1.

Moreover, due to the static nature of some networks, many resource allocation and incentive mechanism design works assume that the supply and demand of resources are fixed. However, as the network evolves, the demand for network resources can be highly fluctuating, and immediate supply of additional resources is required during peak hours. Thus, it is critical to consider the variability of heterogeneous networks and the uncontrolled nature of individuals on both sides of networks. Under the one-sided market model, a network operator is unable to balance supply and demand simultaneously. Such information asymmetry in a one-sided market leads to transaction costs, that is, system inefficiency in the networks. As a result, the obtained resources might exceed or be insufficient compared to the demand, resulting in resource under-utilization or shortage. Thus, the resource efficiency under current designs of the one-sided market model still has room for improvement.

Facing the challenges of the future network, there is a need for an efficient means to balance demand and supply, that is, dynamically and simultaneously provide incentives to the resource owner and allocate the resources to end users in a more flexible way. Thus, we intend to build a resource sharing platform based on the two-sided market model, which integrates demand and resources as the demand pool and the supply pool, then bridges the users and suppliers to break down information barriers. Under a two-sided market model, the network operator must choose the right strategy toward groups on each side of the market, so that the demand and supply are balanced to achieve higher profit or efficiency. Evolving from the one-sid-

Yanru Zhang is with the University of Electronic Science and Technology of China; Yingjie Zhou (corresponding author) is with Sichuan University; Zhi Liu is with Shizuoka University; Bidushi Barua is with the University of Oulu;

Duy H. N. Nguyen is with San Diego State University. 


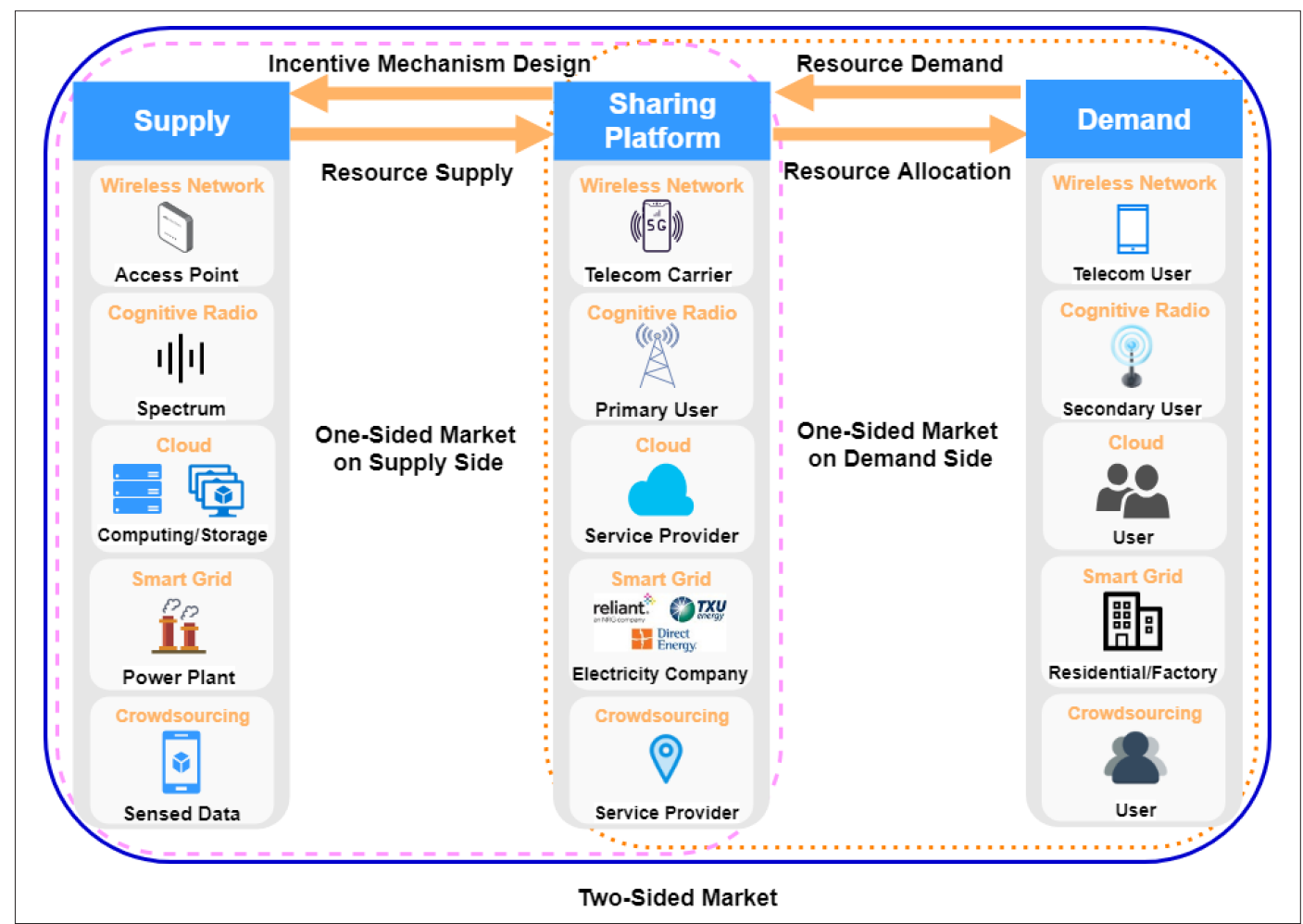

FIGURE 1. Resource allocation on the demand side and incentive mechanism design on the supply side in wireless networks and Internet.

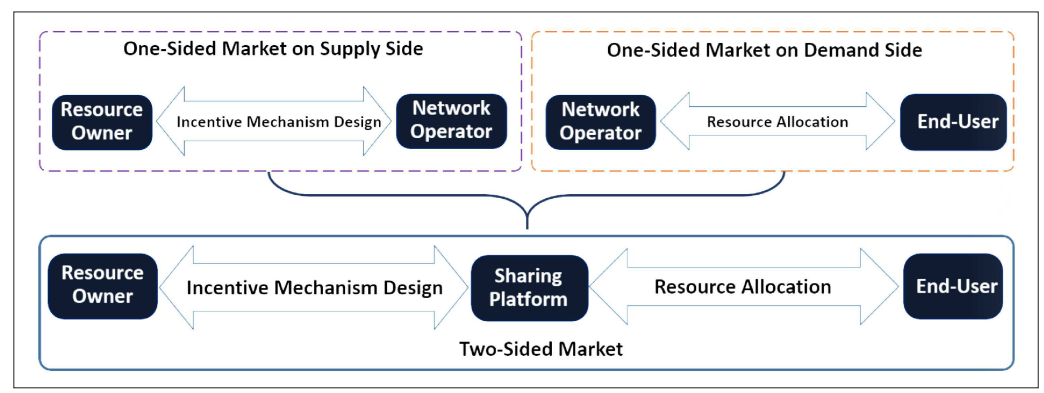

FIGURE 2. The evolving of resource sharing model from one-sided market to two-sided market. of resources can increase not only for the business and individuals, but also for the community and society [2].

In the rest of the article, we will start with the basic concept and challenges of the two-sided market model. Then we proceed to the applications of the two-sided market in various networks. The potential methodologies to solve resource sharing problems in a two-sided market are also presented and compared sequentially. In the end, the future directions for resource sharing in a two-sided market are discussed.

\section{Two-Sided Marke:: Concept and Challenges}

In this section, the basic concept of the two-sided market model will be presented first, followed by the current trend in the sharing economy which is one of the typical applications of the two-sided market. In the end, the challenges of adopting a two-sided market to facilitate resource sharing in wireless networks will be introduced.

BASIC CONCEPT
A two-sided market, also known as a two-sided two-sided market model can be found in communication networks and Internet, such as Uber and Airbnb, which share idle resources with traditional service offerings. Other representative two-sided market business models include credit cards (composed of cardholders and merchants), operating systems (end-users and developers), video-game consoles (gamers and game developers), and search engines (advertisers and users). A two-sided pricing strategy generally increases the platform's total profit compared to a traditional one-sided strategy, as we are able to see the huge market value of Uber compared to the traditional taxi company. Through the exchange of information about resources through a sharing platform, the value network, is an economic network with two independent groups of players who provide network benefits to each other. Both sides of the market have costs and benefits, while platform(s) that connect the two sides of the market face the problem of how to balance supply and demand by adopting specific strategies based on different network characteristics and objectives. The efficiency of a two-sided market is severely limited by the imbalance between supply and demand, and can be regulated and controlled by a platform through pricing and other means. For example, the sharing platform can send signals to the supply side regardless of whether the resources 
are scarce or redundant, motivating the resource owners to adjust the amount of resources to be provided, then narrow the gap between supply and demand, and improve the efficiency of the entire system.

\section{SHARING ECONOMY}

The sharing economy is a popular business model based on strangers and the temporary transfer of the right to use resources, the essence of which is the integration of idle resources. Nowadays, the common sharing economy business model mainly exists in the fields of traffic (Uber), accommodation (Airbnb), communication (WiFi Hotpot), and finance (P2P). According to the operation mode, the business model of the sharing economy can be further divided into the business to the customer (B2C) model (sharing a bicycle, power bank) and customer to customer (C2C) model (Uber, Didi, and Airbnb).

The pricing strategy plays a significant role in the sharing economy and two-sided market. As one of the unicorn companies in the field of the sharing economy, Uber has received a lot of attention due to its real-time pricing model, which is still unrevealed by Uber. A large number of works have tried various ways such as machine learning, queuing theory, and black box to reverse engineer Uber's dynamic pricing strategy, as it can potentially be utilized in other two-sided market businesses [3]. In order to capture the market and ensure the full supply of drivers, Uber has implemented various concessions and subsidies for customers and drivers, and consumed cash at an incredible rate. Thus, the subsidy design in the sharing economy is also the key strategy that affects the efficiency and profit of the platform. One example is [4] which designs an efficient subsidy mechanism for drivers by proposing a "frequent flyer plan" for drivers, by which drivers are encouraged to serve on the platform for a long time, instead of moving between different platforms.

\section{CHALENGES}

The structure and network effects of the two-sided market model result in unique challenges compared to the one-sided market model.

Deal with the Network Effects: In a two-sided market, there are two types of network effects which are tangled with each other and must be carefully dealt with to facilitate the operation of the system [1]. One type is same-side network effects, where the strength of one side has an impact on its growth. It can be positive, for example, the user experience on a social media network gets better with more users on it. It can also be negative: a cloud service provider will be less attractive to merchandisers when all their competitors have already saturated the market. The other type is cross-side network effects, when the strength of one side has an impact on the growth of the other. They can be positive: the more users that a service provider has gathered, the more attractive it is to content and resource providers. they can also be negative: the more users there are in a wireless network, the less attractive it is to potential users due to the congestion effect.

Balance the Demand and Supply: Due to the dynamic and heterogenous features of wireless networks, the demand and supply of resources fluctuate rapidly with time and place. The demand of end users is typically elastic, whereas the resources purchased or leased from resource owners are fixed-term. Thus, adjusting supply as user demand changes is significantly important for the system efficiency. Together with the unique structure of the two-sided market model, the network faces a high degree of uncertainty in the availability of resource supply, which is more challenging compared to the one-sided market model. Furthermore, with the rapid development of the Internet and the sharing economy, there has been cooperation and competition for users and resources inter and intra sharing platforms, which have aggravated the demand for efficient resource allocation algorithms that can regulate the players to be individually rational, truthful, and budget-balanced as well.

Get the Right Price: To facilitate users and resource owners' interactions with network effects, the platform is in need of a set of rules, such as protocols and pricing strategies, that govern transactions and factor in the impact on the other side's growth and willingness to pay. However, managing the platform now becomes tricky, since the strategies that make traditional offerings successful will not work in these two-sided markets.

Security the Privacy: The accurate matching between supply and demand typically involves the publication or the exchange of personal information, which requires some implicit or explicit privacy considerations by resource owners and users. Because of its inherent particularity, the two-sided market model challenges the suitability of existing information security and privacy protection methods in the one-sided market model.

\section{Two-Sided Market Applications in WIRELESS NETWORKS AND THE INTERNET}

With the rapid development of communication and computer networks, the requirements of higher data rates and lower latency, as well as the need for ubiquitous connectivity as the Internet of Things sector emerges, can significantly exceed the current network capacity and in highly demand of network resources to accommodate future services. Therefore, the imminent shortage of resources provides new impetus for identifying practical solutions to make the most efficient use of resources in a shared manner. Resource sharing in wireless networks and the Internet can be regarded as a sharing economy business form that provides resource owners and end users with a platform that enables the efficient optimization of resources through the mutualization of excessive capacity in resources and services. The two-sided market model provides an efficient means to balance resource demand and supply, that is, simultaneously providing an incentive to the resource provider and allocate the resource to the demander for future networks. The two-sided market model is promising to leverage resource sharing efficiency in heterogeneous networks and the Internet. However, there are quite a few studies that have adopted the two-sided market model compared to the rich literature based on the one-sided market model. In this section, seminal works in wireless networks and the Internet that have allocated resources in a shared manner with a two-sided market model will be introduced, such as cloud,
A two-sided market, also known as a two-sided network, is an economic network with two independent groups of players who provide network benefits to each other. Both

sides of the market

have costs and benefits,

while platform(s) that connect the two sides of the market face the problem of how to balance supply and demand by adopting specific strategies based on different network

characteristics and objectives. 
In cloud trading mar-

kets, the resource

owners sell various

resources such as soft-

ware and infrastructure

with specific features

and functionalities

through a cloud service

platform. Both users

and resource owners

in the market want to

maximize their own

utilities, and pricing is

the key for the plat-

form to balance the

demand and supply.
Internet access point, spectrum, and smart grid transmission line shown in Fig. 1.

\section{RESOURCE TRADING IN THE CLOUD}

In cloud trading markets, the resource owners sell various resources such as software and infrastructure with specific features and functionalities through a cloud service platform. Both users and resource owners in the market want to maximize their own utilities, and pricing is the key for the platform to balance the demand and supply. For example, one company can offer artificial intelligence (AI) and machine learning $(\mathrm{ML})$ services to users without owning an infrastructure. The company can get the cloud computing pool from a cloud service provider, which significantly lowers the capital expenditure. The work in [5] models the trading of virtual machines between users and resource suppliers through the cloud platform. Specifically, the authors formulate the resource transactions as a double auction, in which both suppliers and users submit their sell-bids and buy-bids to the platform, respectively. Then the platform matches the winner among the suppliers and users according to their bids, which can effectively balance the suppliers' resources and users' task requirements.

\section{INTERNET ACCESS FROM SERVICE ProvideRS}

Internet service providers (ISPS) serve as a platform that connects end users (EUs) and content providers (CPs) for data exchange in a two-sided market. Some ISPs have announced their intention to charge both CPs and EUs to cover the investment in network capacity and operation cost, and provide differential allocation of connectivity parameters based on the payment received [6]. The single ISP's profit maximization problem, where EUs and CPs are charged for interconnection is covered by [7]. The more general problem where there are multiple ISPs existing in the market to share profit is considered by [8]. specifically, the authors model the noncooperative interaction between different ISPs as a Stackelberg game and quantifying the resulting loss in social profit due to competition.

\section{INFRASTRUCTURE SHARING IN WIRELESS NETWORK VIRTUALLATION}

Virtualization of wireless mobile networks has become the next hot topic following wired network virtualization, which aims at allowing network or infrastructure providers to manage and control their networks in a more dynamic fashion [9]. Wireless mobile network virtualization standing out itself by enabling abstraction and sharing of infrastructure and physical resources, where the virtual network operator (platform) serves mobile users without owning infrastructure, but can request and pay physical mobile network operators in a more pay-as-youuse manner. Through resource virtualization, the expenditure of wireless network deployment and operation can be reduced significantly.

\section{SpeCtRum Sharing In Cognitive NetworkS}

Cognitive radio networks provide the necessary incentives for PUs and SUs to improve spectrum utilization by allowing SUs to access PUs' idle spectrum for data transmission. In Gao's work [10], a matching algorithm is proposed based on PUs' and SUs' requirements of bandwidth, interference, and maximum transmission power, so that the supply and demand sides in the cognitive radio network are matched as a stable pair.

\section{Electric VehicLe (EV) Charging in SMart Grid}

A collaborative charging strategy for power companies (PCs) to allocate charging stations (CSs) to electric vehicles (EVs) can lead to an optimized solution that considers both the EV user experience and the profits of CSs. The work in [11] proposes a charging navigation system that combines the power network with the transportation network to provide real-time navigation for EVs, attracting EVs to charge during off-peak hours, saving customer waiting time, so as to balance the supply and demand of the charging station and improve the profit of the power system.

\section{METHODOLOGIES TO Solve Two-Sided Market Problems}

Among the seminal works that have modeled the system as a two-sided market, several approaches have shown great potential in solving the problem. In the following subsections, we will introduce several methodologies that have already been proved or have the potential to provide a quite general characterization of the two-sided market in a wide range of complicated networks.

\section{MATCHING THEORY}

Two-sided markets aiming at finding the optimal and stable matching between supply and demand. In such markets, the Nobel-Prize-winning matching theory can not only systematically capture the cooperative interaction between the two individuals on both sides, but also systematically capture the competitive interaction between the individuals on one side. The classic two-sided market model was proposed by Gale and Shapley [12] about the stable matching of marriage, and has been approved high practicality in kidney exchange and college admission. The work in [10] has adopted matching theory to solve the cooperative spectrum sharing problem in cognitive networks under incomplete information. The work in [13] solves the problem of cooperative communication in wireless networks by analyzing the transmission characteristics and requirements of source nodes and relay nodes to accurately match them based on their preference.

\section{StACKELBERg GAME}

The Stackelberg game is a strategic game in which at least one player is defined as a leader who can make decisions and propose strategies before other players are defined as followers, that is, the leader moves first and then the followers move sequentially. Stackelberg games have contributed much in the security domain, supply chain, and marketing channels. The work in [8] examines the issue of inter-platform competition for revenue and rate allocation in the presence of multiple ISP platforms. In the absence of collusion between platforms, each selfish ISP intends to maximize its own profits, which inevitably leads to the loss of social welfare. The authors try to reduce the loss of social welfare by simulating the non-cooperative interaction between the "eyeball" ISP and the "content" ISP as the Stackelberg 


\begin{tabular}{|l|c|c|c|}
\hline Methodology & $\begin{array}{c}\text { Mutual- } \\
\text { ack }\end{array}$ & $\begin{array}{c}\text { Multi- } \\
\text { platform }\end{array}$ & $\begin{array}{c}\text { Multi- } \\
\text { service }\end{array}$ \\
\hline Matching theory & Yes & No & Yes \\
\hline Stackelberg Game & No & Yes & Yes \\
\hline Double auction & No & No & Yes \\
\hline Queuing theory & No & Yes & Yes \\
\hline
\end{tabular}

TABLE 1. Summary of methodologies for resource sharing in two-sided market.

game. However, many existing works that adopt the Stackelberg game to tackle the two-sided market problem consider only the static case. For the case where the demand and supply fluctuate frequently which requires real time and dynamic analysis, the dynamic Stackelberg game is yet to be used to resolve these problems.

\section{DOUBLE AUCTION}

Auction theory has been widely used for solving resource allocation problems since it can overcome asymmetries in information flow and for allocation resource efficiently based on players' truthful bids. In many existing works, authors typically assume that there is only a single resource supplier, and users cannot choose to purchase resources from multiple vendors to achieve greater cost efficiency. However, in many practical scenarios, multiple suppliers often co-exist in a market. For example, in cloud computing, cloud service providers integrate cloud resources from different suppliers, and provide users with a unified application programming interface (API) to access cloud resources. Since different suppliers have different pricing models and users have different task requirements, it is difficult for both parties to make the best decisions. The double auction is an effective method to balance the interests of both cloud providers and users. In particular, the auctioneer collects bids from all potential buyers and sellers. Then the auctioneer announces a market clearing price: all sellers whose bids are lower than the clearing price are sold at this price. All buyers whose bids exceed the clearing price are bought at this price, and the supply and demand are balanced at the moment. In the mechanism proposed by [5], the cloud integration platform can be regarded as an auctioneer who determines the price and quantity of cloud resources and the winners of both parties after collecting the bids of all users and cloud suppliers.

\section{QUEUING THEORY}

Queuing theory is a mathematical study of waiting queues. By constructing a queuing model, the queue length and waiting time can be predicted. Queuing theory has been widely used in transportation systems, port berth design, machine maintenance, inventory control and other service systems, such as shops, hospitals, and so on, and also in the fields of telecommunications and computing. In a two-sided market, the sharing platform can be modeled as the server, and the resource demanders are regarded as the tasks to be handled. The server is responsible for allocating the tasks so that the system can achieve the maximum efficiency. Queueing theory can not only model the two-sided market and capture the incentives of both players on both sides of the platform, but also satisfy the stochastic feature and capture the dynamics of players in the system. Thus, queueing theory has been recently used to study the dynamic pricing strategies in ride-sharing platforms, such as the work in [14] which has adopted a two-queue example of an open Jackson network and tries to reveal the surge pricing of Uber by modeling the passenger queueing process based on real-time state, which influences the transaction volume and profit of the platform. In [11], the authors modeled the EVs charging queueing process as an $\mathrm{M} / \mathrm{M} / \mathrm{C}$ queue with $c$ identical servers, in which each EV and charging station can be viewed as the task and server, respectively. However, the current application of queueing theory for the two-sided market problem in the resource sharing area is still rare to see.

\section{COMPARISON AMONG METHODOLOGIES}

Giving the aforementioned works, here we discuss and compare the pros and cons of adopting those methodologies to solve resource sharing problems in a two-sided market. When using matching theory to solve the two-sided market problem, one shortcoming is that it requires both sides of players to have a complete or at least part of the information of all players on the other side. For example, the match between students and universities is based on their mutual understanding through research and interview, so that each side of the players can generate a preference list of the players on other side. After the players on each side submit their preference lists, the platform can generate stable matching pairs based on certain objective and matching algorithms. Due to this property, matching theory shows outstanding performance in applications in practice such as college admissions, and in wireless networks such as spectrum trading, but may not be practical for scenarios in which players on one side do not have full research of the players on the other side of the market. However, it is quite general for users not knowing the complete information of resource providers in cloud trading, communication networks, and so on. The Stackelberg game shows great potential in solving two-sided market problems, not only with single platform only cases, but also for the case where there are multiple platforms with competition. Double auction theory is highly capable of solving two-sided market resource sharing. However, since there is only one auctioneer allowed, auction theory is unable to solve the inter platform competition problem due to the non-existence of multi-auctioneer model.

Some key properties of the above methodologies are summarized in Table 1. Matching theory is the only model that requires players on one side to receive acknowledgment from the players on the other side, so as to generate a preference list before the matching process starts. Furthermore, the case of multiple platforms is not supported in the matching and auction model, and thus cannot be used to study inter-platform competition problem in the two-sided market. Luckily, all four methods are able to model the case where there
The Stackelberg game is a strategic game in which at least one player is defined as a leader who can make decisions and propose strategies before other

players are defined

as followers, that is,

the leader moves first and then the followers

move sequentially.

Stackelberg games have contributed much in the security domain, supply chain, and marketing channels. 


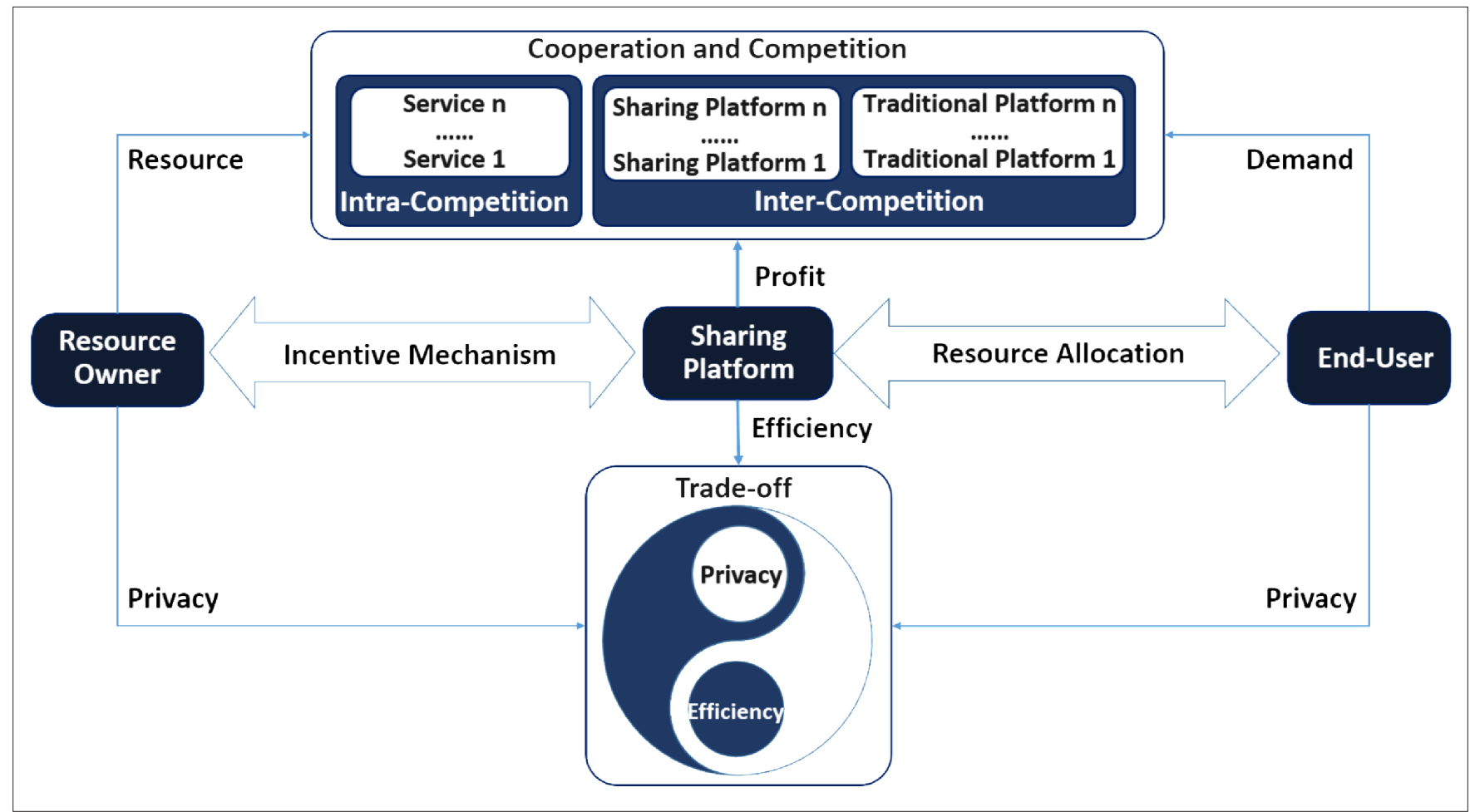

FIGURE 3. Future directions.

are multiple services offered by a platform, which is promising to solve the intra-competition within platform.

\section{FUTURE DIRECTIONS}

The previous sections present the basic background of the two-sided market, and the potential methodologies to solve resource sharing under such a model. In this section, we are going to discuss several future directions of resource sharing under the two-sided market model which are also summarized in Fig. 3.

\section{RESOURCE SHARING BASED ON THE TWO-SIDED MARKET MODEL}

Facing the rapid development of wireless networks to provide various broadband services, there is an imminent demand for dynamic sharing of idle resources to cover the high cost of capital expenditures. Given the large body of works based on the one-sided market model in areas such as spectrum sharing in cognitive radio network, network access point sharing in cellular network, power line sharing in smart grid, and so on, there are many works to be done by switching to the two-sided market model and improve the current performance based on one-sided market model.

\section{COLlaboration and COMPEITION BASED ON MULTI-PLATFORM AND MULII-SERVICE IN TwO-SIDED MARKET}

Most existing works on resource sharing only consider the monopoly market model, that is, assuming that there is only one platform and one service in the market, ignoring the fact that multiple platforms often exist in practical applications, and one platform provides multiple services at the same time. Therefore, there exists competition for resources and users among different sharing platforms and different services within the same platform. The collaboration and competition issues in inter and intra platforms are commonly seen in the real world with multi-platform and multi-service models, and much work needs to be done [15].

\section{Trade-Off Between the EFFICIENCY OF THE Sharing PlatForm AND the PrIIACY OF Users}

Under the structure of the two-sided market, the platform faces the challenge of how to efficiently match supply and demand. Intuitively, the more private information of users is disclosed and exchanged, the higher probability that the platform can successfully match demand with supply. However, more exposure means higher risk, such as data leakage and identity theft. Therefore, the platform faces the trade-off between higher efficiency and profit, and the protection of user privacy. There is also a game of interest between users and platforms. It is interesting to address what privacy trade-offs could take place in exchange for users' participation, how it affects their willingness to participate, and the benefits they derive from their participation. Such a unique structure of the two-sided market leads to new challenges for privacy protection, resulting in the failure of existing methods, since most of research is based on the one-sided market model. Indeed, privacy concerns have been identified as some of the main factors for participating, or not, in resource sharing. The research on user privacy protection in the two-sided market has not been given much attention yet. Therefore, combing the special structure of the two-sided market to open new research in the field of user privacy protection has important meaning in resource sharing. 


\section{CONCLUSIONS}

We have studied how to use a two-sided market model to solve resource sharing in wireless networks and Internet based on the existing works on each side of the market: the incentive mechanism design between resource owners and network operator, and resource allocation between network operator and end users. In particular, the basic concept and inherent challenges of the two-sided market are provided sequentially, and the applications of using the two-sided model are further discussed to solve resource sharing in typical wireless networks and the Internet. The potential methodologies to solve resource sharing problems in the two-sided market are also presented sequentially. In the end, the future directions for resource sharing in the two-sided market are discussed. Overall, there exists a variety of resource sharing problems in wireless networks and the Internet whose efficiency can be significantly improved by adopting a two-sided market model.

\section{ACKNOWLEDGMENT}

The work of Yingjie Zhou is partly supported by the National Natural Science Foundation of China (NSFC) with grant number 61801315.

\section{REFERENCES}

[1] J.-C. Rochet and J. Tirole, "Two-Sided Markets: A Progress Report," The RAND J. Economics, vol. 37, no. 3, 2006, pp. 645-67.

[2] J. Hamari, M. Sjöklint, and A. Ukkonen, "The Sharing Economy: Why People Participate in Collaborative Consumption," J. Association for Information Science and Technology, vol. 67, no. 9, 2016, pp. 2047-59.

[3] Z. Fang, L. Huang, and A. Wierman, "Prices and Subsidies in the Sharing Economy," Proc. Int'l. Conf. World Wide Web (WWW), 2017, pp. 53-62.

[4] Z. Fang, L. Huang, and A. Wierman, "Loyalty Programs in the Sharing Economy: Optimality and Competition," Proc. Eighteenth ACM Int'l. Symposium on Mobile Ad Hoc Networking and Computing (MobiHoc), 2018, pp. 131-40.

[5] L. Lu et al., "A double Auction Mechanism to Bridge Users' Task Requirements and Providers' Resources in Two-sided Cloud Markets," IEEE Trans. Parallel and Distributed Systems, vol. 29, no. 4, Apr. 2018, pp. 720-33.

[6] G. Kesidis, "A Simple Two-Sided Market Model with Side-Payments and ISP Service Classes," Proc. IEEE Conf. Computer Commun. (INFOCOM) Workshops, Apr. 2014, pp. 595-97.

[7] P. Hande et al., "Network Pricing and Rate Allocation with Content Provider Participation," Proc. IEEE Conf. Computer Commun. (INFOCOM), Apr. 2009.

[8] Y. Wu et al., "Revenue Sharing among ISPs in Two-Sided Markets," Proc. IEEE Conf. Computer Commun. (INFOCOM) Workshops, Apr. 2011, pp. 596-600.

[9] C. Liang and F. R. Yu, "Wireless Network Virtualization: A Survey, Some Research Issues and Challenges," IEEE Commun. Surveys Tutorials, vol. 17, no. 1, First Quarter 2015, pp. 358-80.

[10] L. Gao, L. Duan, and J. Huang, "Two-Sided Matching Based Cooperative Spectrum Sharing," IEEE Trans. Mobile Computing, vol. 16, no. 2, Feb. 2017, pp. 538-51.

[11] J. Tan and L. Wang, "Real-Time Charging Navigation of Electric Vehicles to Fast Charging Stations: A Hierarchical Game Approach," IEEE Trans. Smart Grid, vol. 8, no. 2, Mar. 2017, pp. 846-56.

[12] D. Gale and L. S. Shapley, "College Admissions and the Stability of Marriage," The American Mathematical Monthly, vol. 69 , no. 1,1962 , pp. 9-15.

[13] D. Li et al., "Distributed Relay-Source Matching for Cooperative Wireless Networks Using Two-Sided Market Games," Proc. IEEE Global Telecommunications Conf. (GLOBECOM), Nov. 2009.

[14] S. Banerjee, C. Riquelme, and R. Johari, "Pricing in RideShare Platforms: A Queueing-Theoretic Approach," IEEE Conf. Computer Commun. (INFOCOM) Workshops, Feb. 2015.
[15] S. Shakkottai and R. Srikant, "Economics of Network Pricing with Multiple ISPs," IEEE/ACM Trans. Networking (ToN), vol. 14, no. 6, Dec. 2006, pp. 1233-45.

\section{BIOGRAPHIES}

YANRU ZHANG [S'13, M'16] received the B.S. degree in electronic engineering from the University of Electronic Science and Technology of China (UESTC) in 2012 and the Ph.D. degree from the Department of Electrical and Computer Engineering, University of Houston in 2016. From 2017 to 2018, she was a post-doctoral fellow with the Information Engineering Department, the Chinese University of Hong Kong. She is currently a professor with the School of Computer Science and Engineering, UESTC. Her current research involves resource allocation and mechanism design with contract theory and matching theory in the Internet and applications, wireless communications and networking. She received the Best Paper Awards at IEEE ICCS in 2016 and IEEE ICCC in 2017.

YINGJIE ZHOU [M'14] received his Ph.D. degree from the School of Communication and Information Engineering, University of Electronic Science and Technology of China (UESTC), China, in 2013. He is currently an assistant professor in the College of Computer Science at Sichuan University (SCU), China. He was a visiting scholar in the Department of Electrical Engineering at Columbia University, New York. His current research interests include network management, behavioral data analysis, and resource allocation.

ZHI LIU [S'11, M'14] received the B.E. from the University of Science and Technology of China, China and Ph.D. degree in informatics from the National Institute of Informatics. He is currently an assistant professor at Shizuoka University. He was a junior researcher (assistant professor) at Waseda University and a JSPS research fellow at the National Institute of Informatics. His research interest includes video network transmission, vehicular networks and mobile edge computing. He was the recipient of the IEEE StreamComm 2011 Best Student Paper Award, the 2015 IEICE Young Researcher Award, and the ICOIN 2018 Best Paper Award. He is and has been a guest editor for journals including Wireless Communications and Mobile Computing, Sensors and the IEICE Transactions on Information and Systems. $\mathrm{He}$ has been serving as the chair for number of international conferences and workshops. He is a member of IEEE and IEICE.

BIDUSHI BARUA received the B.Eng. degree in electronics and telecommunications engineering from the University of Gauhati, India, in 2007, the M.Eng. degree in telecommunications engineering from the Asian Institute of Technology, Thailand, in 2012, and the Dr.Sc. (Tech.) degree in wireless communications engineering from the University of Oulu in 2018, with a focus on techniques of incentivizing user cooperation in cooperative content delivery for wireless networks. She was an assistant manager with Reliance Communications, India, from 2007 to 2008, and with Dishnet Wireless Ltd., India, from 2008 to 2010. She is currently a post-doctoral researcher with the Centre for Wireless Communications, University of Oulu. Her research interests include device-to-device communications, mobile clouds, smart grids, micro-operator networks, contractual agreements between operators, game theory, and contract theory.

DUY H. N. NGuYEN [S'07, M'14, SM'19] received the B.Eng. degree (Hons.) from Swinburne University of Technology, Hawthorn, VIC, Australia, in 2005, the M.Sc. degree from the University of Saskatchewan, Saskatoon, SK, Canada, in 2009, and the Ph.D. degree from McGill University, Montréal, QC, Canada, in 2013, all in electrical engineering. From 2013 to 2015, he held a joint appointment as a research associate with McGill University and a post-doctoral research fellow with the Institut National de la Recherche Scientifique (INRS), Université du Québec, Montréal, QC, Canada. He was a research assistant with the University of Houston in 2015 and a post-doctoral research fellow with the University of Texas at Austin in 2016. Since 2016, he has been an assistant professor with the Department of Electrical and Computer Engineering, San Diego State University, San Diego, CA, USA. His current research interests include resource allocation in wireless networks, signal processing for communications, convex optimization, game theory, and machine learning. He was a recipient of the Australian Development Scholarship, the FRQNT Doctoral Fellowship and Post-doctoral Fellowship, and the NSERC Post-doctoral Fellowship. He has been serving as a TPC member for flagship IEEE conferences, including ICC, GLOBECOM and INFOCOM. He currently serves as an associate editor for the EURASIP Journal on Wireless Communications and Networking.
Privacy concerns have been identified as some of the main factors for participating, or not, in resource sharing.

The research on user privacy protection in the two-sided market

has not been given much attention yet.

Therefore, combing the special structure of the two-sided market to open new research

in the field of user privacy protection has important meaning in resource sharing. 